\title{
Uso de anillo en bypass gástrico: Ventajas y desventajas*
}

\author{
Drs. LUIS F. EVANGELISTA ${ }^{1}$, JOSEMBERG M. CAMPOS ${ }^{1}$, ÁlVARO A. B. FERRAZ ${ }^{1}$, \\ ALEX ESCALONA ${ }^{1}$, MANOEL GALVÃO NETO ${ }^{1}$, ALMINO C. RAMOS ${ }^{1}$, EDMUNDO M. FERRAZ ${ }^{1}$
}

Servicio de Cirugía General, Hospital das Clínicas, Universidade Federal de Pernambuco, Recife; Gastro Obeso Center, São Paulo, Brasil.

\section{Use of ring in gastric bypass surgery: Advantages and disadvantages}

\section{Introducción}

Entre las diversas técnicas operatorias empleadas en el tratamiento de la obesidad, el bypass gástrico en $\mathrm{Y}$ de Roux es el más realizado․ Esta cirugía asocia principios de restricción en la ingesta de alimentos y de malabsorción, logrando adecuada pérdida de peso a largo plazo, con bajo índice de complicaciones y de efectos adversos tardíos ${ }^{2}$.

En 1966, Mason describió el bypass gástrico ${ }^{3}$, el cual fue perfeccionado posteriormente por otros autores $^{4,5}$. Entre las décadas de 1980 y de 1990, Fobi y Capella publicaron series iniciales de pacientes sometidos al bypass gástrico en $\mathrm{Y}$ de Roux con una prótesis de contención, que funcionaba como un factor de restricción al vaciamiento de la bolsa gástrica ${ }^{6,7}$.

El anillo de contención comenzó a ser usado en el bypass gástrico en la década de 1980. Linner aplicaba un refuerzo en la anastomosis gastroyeyunal para prevenir su dilatación ${ }^{5}$. Enseguida, otros autores fueron incorporando y perfeccionando este principio de contención hasta alcanzar el formato del procedimiento quirúrgico actual.

No hay uniformidad en el tipo de material empleado y ni en el tamaño de la circunferencia de la prótesis, siendo descrito el uso de un anillo de silicona o de una cinta de Prolene ${ }^{\circledR}$ o de Goretex $\AA$, cuyo largo varía de 5,5 a $7 \mathrm{~cm}^{6-9}$.

Además, el uso de material protésico en la vía de salida de la bolsa gástrica permanece hasta hoy como un tema controvertido, considerando el real beneficio obtenido en la pérdida de peso y en su mantención a largo plazo, en detrimento del potencial riesgo de ocurrir intolerancia alimentaria, aparte del surgimiento de posibles complicaciones relacionadas al anillo, tales como: migración o erosión intragástrica, deslizamiento y estenosis de la vía de salida del pouch.

Así, los autores objetivan describir las principales ventajas y desventajas del uso del anillo en el bypass gástrico en Y de Roux, a partir de la realización de una revisión de la literatura mundial.

\section{Metodología}

Fueron evaluados artículos científicos publicados en revistas médicas indexadas, a partir de la utilización del sistema de búsqueda de los principales bancos de datos de internet, tales como: Medline, Lilacs, Pubmed y Scielo. Fueron incluidos también capítulos de libros específicos sobre ciru-

\footnotetext{
*Recibido el 12 de Agosto de 2009 y aceptado para publicación el 22 de Septiembre de 2009.

Correspondencia: Dr. Josemberg M. Campos

Rua Vigário Barreto, 127 / 802 - Graças, 52020-140, Recife, Brasil.

E-mail: berg@elogica.com.br
} 
gía bariátrica, que presentaban determinados aspectos aún no publicados en la literatura mundial.

\section{Cuestionamientos}

Los defensores argumentan que el uso del anillo en el bypass gástrico promueve un mayor control del peso a medio y largo plazo. Por otro lado, algunos cirujanos no utilizan la prótesis temiendo una mayor aparición de vómitos y regurgitación, lo que dificulta la ingestión de una dieta adecuada, que puede comprometer el estado nutricional.

\section{¿El uso del anillo en el bypass determina una mayor pérdida de peso?} pico.

Hay pocos artículos prospectivos sobre este tó-

En 2005, Awad et $\mathrm{al}^{10}$, publicó un estudio prospectivo en el primer congreso latinoamericano de la IFSO, comparando 83 pacientes operados de bypass gástrico sin anillo, contra 109 pacientes sometidos a bypass gástrico con anillo; donde no hubo diferencia en la pérdida de peso después del seguimiento posoperatorio de 6,9 y 12 meses. Sin embargo, después de 18 y 24 meses, hubo una diferencia significativa en la disminución del índice de masa corporal (IMC). En el grupo con anillo, la media del IMC disminuyó en 38,5 y 43\% a los 18 y 24 meses respectivamente, mientras que el grupo sin anillo disminuyó el IMC en promedio $29,7 \%$ a los 18 meses y $26 \%$ a los 24 meses.

Similar al estudio anterior, en 2007, Bessler et $\mathrm{al}^{11}$, mostró los resultados iniciales de 90 pacientes superobesos (IMC $>50 \mathrm{~kg} / \mathrm{m}^{2}$ ) sometidos a bypass gástrico, haciendo comparación en los grupos con y sin anillo. Los datos fueron expresados en porcentaje de pérdida del exceso de peso y el seguimiento fue de 36 meses. Después de 6, 12 y 24 meses, no hubo diferencia en la pérdida de peso entre los dos grupos, sin embargo, a los 36 meses, el grupo con anillo alcanzó el mejor resultado (73,4\% contra $57,2 \%$ ), con diferencia significativa.

En 2008, Awad et $\mathrm{al}^{12}$, publicó la mayor serie conteniendo 438 pacientes. Fue un estudio prospectivo que separó grupos de pacientes con características semejantes, que fueron sometidos a bypass con o sin anillo. Tampoco hubo una diferencia significativa de la pérdida del exceso de peso en la evaluación hasta 12 meses. Sin embargo, en las reevaluaciones de 18, 24 y 36 meses, hubo una mayor pérdida del exceso de peso en el grupo con anillo, mostrando una diferencia estadísticamente significativa.

En 2008, Arceo-Olaiz et al ${ }^{13}$, describió el único estudio prospectivo hasta el momento que no mues- tra diferencia en la pérdida de peso entre pacientes operados con o sin anillo. Los 60 pacientes estudiados fueron divididos en 2 grupos y no hubo diferencia significativa en la pérdida de peso, tras 24 meses de seguimiento.

En Brasil, Valezi et $\mathrm{al}^{14}$, publicaron un estudio retrospectivo en que compararon 2 grupos sometidos a bypass con anillo, los cuales fueron divididos de acuerdo con los resultados endoscópicos del diámetro de la compresión extrínseca del anillo en la bolsa gástrica, un año después de la cirugía. El primer grupo presentaba un diámetro interno de hasta $10 \mathrm{~mm}$, y el segundo grupo mostraba un diámetro mayor que $10 \mathrm{~mm}$. La evaluación de 1,2 y 5 años mostró que el grupo con diámetro interno de hasta $10 \mathrm{~mm}$ alcanzó una mayor pérdida del exceso de peso cuando fue comparado al grupo con diámetro mayor que $10 \mathrm{~mm}$.

\section{¿El bypass con anillo promueve una mejor mantención de la pérdida de peso a largo plazo?}

No existe ningún estudio comparativo entre bypass con y $\sin$ anillo que presente resultados más allá de 36 meses. Sin embargo, hay series que muestran un seguimiento tardío, que pueden ser comparadas entre sí.

Buchwald et $\mathrm{al}^{2}$, en un metanálisis bastante citado en la literatura, mostró que la pérdida media del exceso de peso entre 4.204 pacientes sometidos a bypass gástrico sin anillo fue de $61,5 \%$ en dos años. Algunas series de cirujanos que usan exclusivamente la técnica de bypass con anillo muestran una pérdida media del exceso de peso después de 5 años de hasta $83 \% .^{15}$

\section{¿El bypass con anillo causa mayor intolerancia a la ingestión de alimentos?}

En las series que utilizan anillo en el bypass, los autores pasaron a usar prótesis de mayor diámetro basándose en promover una mejora en la tolerancia alimentaria. Stubbs et al, mostró que el uso del anillo de $6,5 \mathrm{~cm}$ de largo determina una mejoría en la calidad de vida y una menor necesidad de retiro del anillo, en comparación al uso del anillo de 5,5 y 6,0 $\mathrm{cm}^{9}$. Salinas et al, también reportó una mejor tolerancia alimentaria al anillo de $6,0 \mathrm{~cm}$, en comparación a la prótesis de $5,5 \mathrm{~cm}^{15}$. Arasaki et al, condujo un estudio mostrando que pacientes con anillo de $6,2 \mathrm{~cm}$ de largo son más propensos a la regurgitación de alimentos, en comparación con los pacientes con anillo de $7,7 \mathrm{~cm}^{16}$.

En los estudios comparativos antes descritos, que también evaluaron la tolerancia alimentaria, hubo una mayor aparición de vómitos en los pacientes con anillo. Sin embargo, los autores ${ }^{13-16}$ afirman que no hubo compromiso de la calidad de vida 
de los pacientes, excepto en los casos de complicaciones asociadas al uso de la prótesis, que serán evaluadas a continuación.

\section{Complicaciones derivadas del uso de anillo en el bypass gástrico}

\section{Migración (erosión) intragástrica de anillo}

La incidencia puede variar de 0,9 a $7 \%{ }^{17-19}$, y la etiología aún no está bien definida, pudiendo ser secundaria a los siguientes aspectos: perforación de la pared gástrica, reacción al cuerpo extraño, anillo apretado, sutura del anillo a la pared gástrica, fístula gástrica y fístula gastrogástrica ${ }^{17,20}$.

El cuadro clínico es inespecífico y variable, siendo el $15 \%$ de los pacientes asintomáticos. El restante puede presentar uno o más de los siguientes síntomas: re-ganancia de peso (37 a $42 \%$ ), dolor epigástrico (18 a 37\%), síntomas obstructivos (13 a $49 \%$ ) y hemorragia digestiva $(8 \text { a } 14 \%)^{17,20,21}$. La endoscopía digestiva alta (EDA) es el mejor medio de diagnóstico, con los siguientes hallazgos: anillo en el interior de la bolsa gástrica, en el yeyuno debajo de la anastomosis o en el asa yeyunal interpuesta (Figura 1) 20-22.

Actualmente, la remoción del anillo migrado ha sido realizada por un método menos invasivo. Así, el procedimiento laparotómico ${ }^{17}$ fue substituido por la endoscopía terapéutica de forma ambulatoria, con sedación moderada y usando los siguientes equipos: endoscopio de un canal, tijera y pinza de cuerpo extraño o asa de polipectomía ${ }^{19,20,22}$. Este tratamiento debe ser precoz e indicado en el momento del diagnóstico, ya que la conducta expectante tiene riesgo potencial de sangrado en la pared gástrica o de impactación alimentaria en el área de la erosión $^{22}$. Así, la remoción del anillo solamente debe ser retrasada cuando haya migración intragástrica insuficiente, lo que dificulta la sección de la prótesis, en tal caso se debe mantener el inhibidor de bomba de protones hasta alcanzar una mayor migración $^{19,20,22}$. El anillo de Marlex también puede ser retirado parcialmente por endoscopía, después de algunas sesiones debido a la mayor adherencia a los tejidos ${ }^{20,22}$.

\section{Deslizamiento del anillo}

Es el deslizamiento distal de la prótesis por fuera de la bolsa gástrica, que puede cabalgar sobre el asa yeyunal y causar dificultad en el vaciamiento gástrico $^{18,19,22-24}$, con una incidencia menor al $1 \% 19,22,23$. El deslizamiento es parcial cuando el endoscopio aún consigue pasar por el área estenótica y total cuando no se logra franquear la obstrucción (Figura 2) 22 .
Las posibles etiologías son: anillo con diámetro mayor que el de la bolsa gástrica, fijación floja del anillo entre la bolsa gástrica y el asa yeyunal interpuesta, posicionamiento inadecuado del anillo en la cirugía y vómitos incoercibles ${ }^{19,25}$.

\section{Diagnóstico del deslizamiento del anillo}

Inicialmente, el anillo está sobrepuesto a la anastomosis gastroyeyunal, y si el deslizamiento distal se intensifica, puede evolucionar hacia una obstrucción total ${ }^{22,25}$.

Deslizamiento parcial: Ocurre aumento progresivo de la frecuencia de los vómitos, eructación, uso sólo de dieta líquida, pérdida de peso, astenia y depresión.

Deslizamiento total: Ocurre pérdida excesiva de peso, vómitos de olor fétido, deshidratación, desnutrición, dificultad de deambulación e intolerancia a cualquier alimento ${ }^{19,25}$.

La radiografía contrastada gástrica puede mostrar lo siguiente: anillo oblicuo o vertical, deslizado en el lado de la fijación al asa interpuesta y en la pared anterior de la anastomosis gastroyeyunal ${ }^{22,24}$. En una fase tardía, el pouch gástrico presenta diámetro aumentado, con nivel hidroaéreo en el fondo gástrico y en el asa interpuesta, anastomosis amplia y anillo oblicuo, promoviendo obstrucción debajo de la anastomosis en la región del asa yeyunal y no visualización del asa interpuesta (Figura 2) 22,25. $^{22}$.

Hallazgo endoscópico: Deslizamiento parcial: área estenótica provocada por el anillo en la anastomosis gastroyeyunal, permitiendo el paso apretado del endoscopio. Deslizamiento total: esofagitis derivada de los vómitos, bolsa gástrica dilatada, formación de fondo gástrico, ausencia de compresión extrínseca en el pouch, residuos alimenticios de olor fétido, anastomosis gastroyeyunal amplia y convergencias de los pliegues yeyunales para la estenosis excéntrica situada debajo o en la región de la anastomosis. En la cara lateral, se produce un pequeño prolapso de la mucosa yeyunal hacia el interior de la bolsa gástrica, causado por la compresión del anillo deslizado (Figura 3) ${ }^{19,22,25}$.

\section{Tratamiento del deslizamiento del anillo}

La conducta específica debe ser precedida por medidas clínicas para la hidratación y reposición hidroelectrolítica. Además, antes de indicarse medidas exclusivas para el control de un posible trastorno alimentario de origen psicológico, se debe considerar la existencia de una posible obstrucción mecánica a través de endoscopía y/o radiografía con contraste yodado.

La conducta clásica ha sido el retiro de la prótesis por laparotomía o laparoscopía ${ }^{18,19,23,25}$. Además, el proceso inflamatorio perigástrico puede dificul- 
tar la cirugía y aumentar el riesgo de fístula, infección y sangrado por lesión hepática o esplénica ${ }^{25}$. Así, existen algunos relatos de tratamiento definitivo menos invasivo a través de la dilatación endoscópica con balón de $30 \mathrm{~mm}$, visando la ruptura del hilo situado en el interior del anillo, que resulta en la apertura de la prótesis y en la mejoría de los síntomas obstructivos $^{22,24,26}$.

Dilatación endoscópica del ani1lo: La técnica es realizada con sedación profunda, debido al uso de balón de gran diámetro, lo que causa incomodidad abdominal en el momento de la insuflación. La descripción de los detalles técnicos es obtenida de libros de endoscopía (Figura 4) $22,24,26$.

La recuperación es inmediata y permite la realización de actividades habituales después de $24 \mathrm{hs}$, sin embargo, el paciente debe ingerir una dieta líquida por 48 hs y usar un inhibidor de bomba de protones ${ }^{24,26}$.

\section{Dificultad de vaciamiento gástrico secundario al anillo}

El estrechamiento en el área de la compresión extrínseca del anillo, en la vía de salida del pouch, retarda el vaciamiento gástrico $\mathrm{y}$ dificulta el paso de un endoscopio de $9,8 \mathrm{~mm}$ de diámetro. En pocos casos, el anillo se encuentra apretado e impide el paso del endoscopio. Además, algunos pacientes no poseen la referida estenosis y también presentan disfagia y vómitos como consecuencia del inadecuado vaciamiento gástrico $0^{9,27,28}$. La intolerancia al anillo, incluso sin presentar deslizamiento o migración, puede indicar su retiro quirúrgico en hasta el $5 \%$ de los casos ${ }^{9}$.

Etiopatogenia posible: Rápida ingesta de alimentos o en mayor cantidad, masticación inadecuada, alteración motora esofágica, bolsa gástrica dilatada encima del anillo y la simple presencia de una prótesis rígida adyacente al trato di-

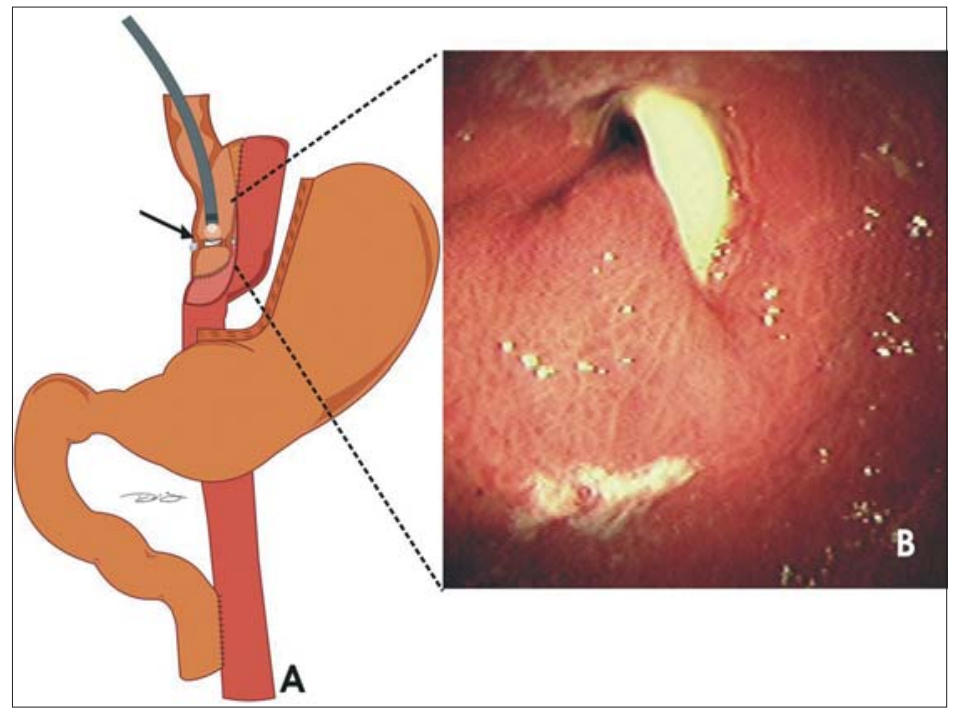

Figura 1. Migración del anillo visualizada en: A) Ilustración de bypass gástrico y B) Hallazgo endoscópico.

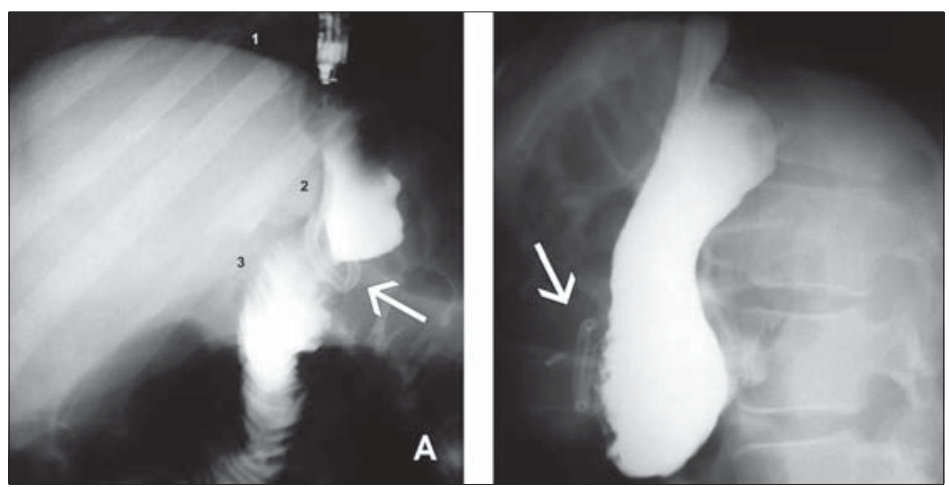

Figura 2. Radiografia de la bolsa gástrica con contraste revelando estenosis gastroyeyunal por deslizamiento del anillo: A) Parcial y B) Total.

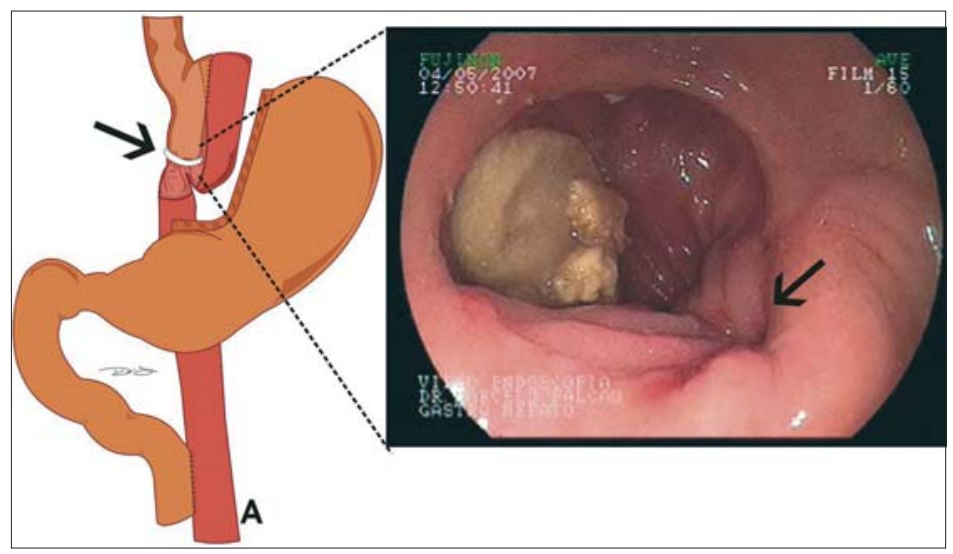

Figura 3. A) Ilustración de un bypass gástrico con deslizamiento del anillo (flecha) y B) Hallazgo endoscópico: Anastomosis amplia, impactación alimentaria y obstrucción debido a la compresión del anillo (flecha). 


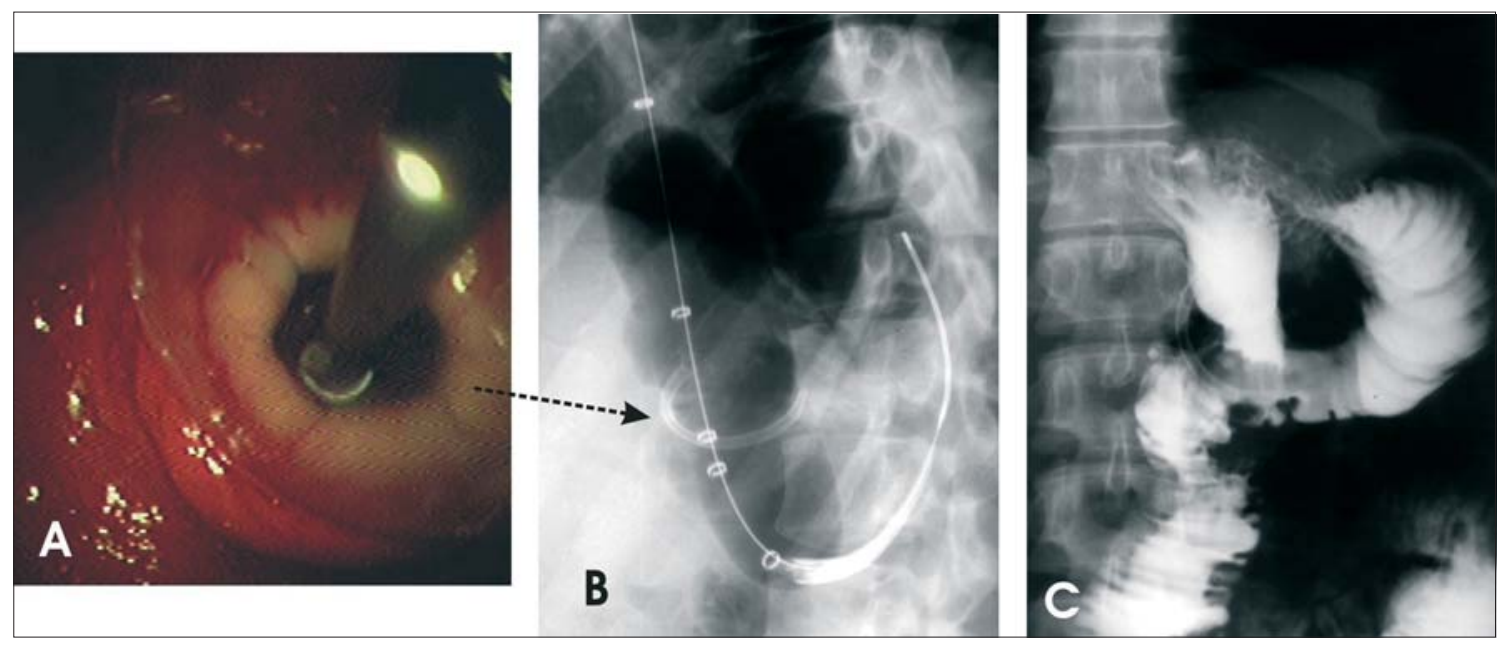

Figura 4. A) Endoscopía: Balón dilatando un anillo (halo emblanquecido); B) Radiografía: Balón causando abertura del anillo (flecha) y C) Radiografía con contraste: Anillo abierto y corrección de la obstrucción gastroyeyunal.

gestivo $^{28}$. Arasaki et al, realizó una correlación entre la hipotonía del esfínter inferior del esófago y la presencia de regurgitación crónica ${ }^{16}$. Cuadro clíni$c o$ : vómito postprandial, sialorrea, disfagia, intolerancia a alimentos sólidos, y tardíamente, pérdida excesiva de peso, deshidratación y desnutrición ${ }^{27,28}$.

Hallazgos endoscópicos: Esofagitis de reflujo, dilatación del esófago y del pouch, estasis alimentaria en el esófago y en el pouch, anillo bien posicionado, anillo apretado, con dificultad para el paso del endoscopio ${ }^{28}$.

Hallazgos radiológicos con contraste: Tránsito esofágico lento, calibre aumentado, presencia de ondas terciarias, pseudo-acalasia. Bolsa gástrica con reservorio dilatado encima del anillo y estrechamiento en la región del anillo 28,29 .

Semejante al deslizamiento del anillo, el tratamiento clásico preconizado ha sido el retiro de la prótesis por laparotomía o laparoscopía, pero también hay la posibilidad de dilatación endoscópica con balón, visualizando la ruptura del hilo interno y la apertura del anillo o el aumento del diámetro de la prótesis $^{22,28}$ (Figura 4).

Los autores realizan este método endoscópico en los pacientes con alteración del vaciamiento gástrico y de deslizamiento del anillo, obteniendo éxito en la mayoría de los casos y con baja morbidad ${ }^{22,28}$. La terapéutica endoscópica está contraindicada en las siguientes situaciones: anillo con formato semejante a la banda gástrica con lacre y sin hilo circular interno y signos clínicos (dolor abdominal) o endoscópicos de necrosis de la pared gastrointestinal (mucosa oscura y olor fétido), con o sin perforación. En estos casos o en las pocas situa- ciones en que ocurre fallo de la dilatación endoscópica, se indica el retiro quirúrgico de la prótesis $^{9,27}$.

\section{Consideraciones finales}

El tratamiento quirúrgico de la obesidad mórbida y principalmente el bypass gástrico, es un abordaje universalmente aceptado, con respaldo de estudios a largo plazo. Sin embargo, el uso del anillo restrictivo en bypass gástrico permanece como un tema controvertido.

Evaluando los datos disponibles en la literatura hasta el momento, no hay muchos estudios comparativos a largo plazo, pero los pocos trabajos parecen mostrar que el bypass con anillo presenta más ventajas en relación a la pérdida de peso a largo plazo y a la mantención en el tiempo en comparación con el bypass sin anillo.

A pesar de esto, incluso los pocos estudios comparativos publicados hasta el momento no son uniformes en sus conclusiones, posiblemente por usar anillos de materiales y circunferencias diferentes. Los estudios de Awad y Bessler ${ }^{10-12}$, utilizaron anillos de 6,0 y $5,5 \mathrm{~cm}$ y encontraron diferencias de pérdida de peso en el seguimiento de 2 años, mientras que Arceo-Olaiz ${ }^{13}$ utilizó $6,5 \mathrm{~cm}$ y no encontró diferencias.

La tolerancia alimentaria es menor en los pacientes sometidos al bypass con anillo cuando es comparada en los pacientes sin anillo ${ }^{10-13}$. Sin embargo, los 4 estudios comparativos y prospectivos encontrados en la literatura hasta el momento fueron en- 
fáticos a la hora de afirmar que estos problemas fueron resueltos de modo no invasivo en los casos de anillo bien posicionado, no alterando la calidad de vida de los pacientes con anillo ${ }^{10-13}$. Apenas un caso en el estudio de Arceo-Olaiz ${ }^{13}$ y 1 en el estudio de $\mathrm{Awad}^{12}$ necesitaron de retirada de la prótesis y esto ocurrió por estenosis y/o deslizamiento de la prótesis ${ }^{10,12}$. La calidad de vida no fue afectada en el resto de los pacientes ${ }^{10-13}$.

Las complicaciones derivadas del uso de la prótesis presentan una frecuencia relativamente baja. Además de esto, el uso de métodos endoscópicos mínimamente invasivos para el tratamiento de estas complicaciones evita re-operaciones y están siendo paulatinamente introducidos en la práctica clínica, como ha ocurrido en la experiencia de los autores $^{20,22,28}$.

Por lo tanto, la decisión del uso del anillo restrictivo debe ser tomada considerando lo siguiente:

- El anillo en el bypass gástrico parece causar beneficio en la pérdida de peso y en su mantención a largo plazo.

- La intolerancia a su uso es alta cuando el anillo tiene un diámetro de $5,5 \mathrm{~cm}$ y aceptable con una circunferencia de $6,0 \mathrm{~cm}$.

- El anillo presenta mayor riesgo de complicaciones y los pacientes deben tener un seguimiento posoperatorio riguroso, siendo indicada la endoscopía en la fase inicial de los síntomas, para realizar un diagnóstico precoz de alteraciones específicas del anillo.

- La migración intragástrica del anillo es de fácil resolución por vía endoscópica.

- El deslizamiento y la estenosis gástrica del anillo habitualmente son solucionados a través de la remoción quirúrgica de la prótesis, pero también pueden ser tratados por dilatación endoscópica con balón para la ruptura del anillo.

\section{Referencias}

1. Buchwald H, Williams SE. Bariatric surgery worldwide 2003. Obes Surg 2004; 14: 1157-1164.

2. Buchwald H, Avidor Y, Braunwald E, Jensen MD, Pories W, Fahrbach K, et al. Bariatric surgery: a systematic review and meta-analysis. JAMA 2004; 292 : 1724-1737.

3. Mason EE, Ito C. Gastric bypass in obesity. Surg Clin North Am. 1967; 47: 1345-1349.

4. Griffen WO Jr, Bivins BA, Bell RM, Jackson KA. Gastric bypass for morbid obesity. World J Surg 1981; 5: $817-822$.

5. Linner JH. Comparative effectiveness of gastric bypass and gastroplasty. Arch Surg 1998; 22: 925-935.

6. Fobi MAL, Lee H, Flemming AW. The surgical technique of the banded gastric bypass. J Obes Weight
Regul 1989; 8: 99-102.

7. Capella RF, Capella JF, Mandec H, Nath P. Vertical banded gastroplasty-gastric bypass: preliminary report. Obes Surg 1991; 1: 389-395.

8. Garrido Jr AB. Cirurgia em obesos mórbidos: Experiência pessoal. Arq Bras Endocrinol Metab 2000; 44: 106-110.

9. Stubbs RS, O'Brien L, Jurikova L. What ring size should be used in association with vertical gastric bypass? Obes Surg. 2006; 16: 1298-1303.

10. Awad W, Garay A, Oñate VH, Turu J. Gastric bypass with and without a ring: the effect on weight reduction and quality of life. Obes Surg 2005; 15: 724-729.

11. Bessler M, Daud A, Kim T, DiGiorgi M. Prospective randomized trial of banded versus nonbanded gastric bypass for the super obese: early results. Surg Obes Relat Dis 2007; 3: 480-484 .

12. Awad W, Garay A, Martínez C, Oñate V, Turu I, Yarmuch J. Descenso ponderal y calidad de vida mediante la cirugía de Bypass gástrico con y sin anillo de calibración. Rev Chil Cir 2008; 60: 17-21.

13. Arceo-Olaiz R, Nayví España-Gómez M, MontalvoHernández J, Velázquez-Fernández D, Pantoja J et al. Maximal weight loss after banded and unbanded laparoscopic Roux-en-Y gastric bypass: a randomized controlled Trial. SOARD 2008; 4: 507-511.

14. Valezi AC, Brito EM, Souza JCL, Guariente ALM, Emori FT, Lopes VCH. A importância do anel de silicone na derivação gástrica em Y-de-Roux para o tratamento da obesidade. Rev Col Bras Cir 2008; 35: 18-22.

15. Salinas A, Salinas BHM, Santiago E, García W, Ferro Q, Antor MM. Silastic ring vertical gastric bypass: cohort study with $83 \%$ rate of 5-year follow-up. SOARD 2008; 5: 455-458.

16. Arasaki CH, Del Grande JC, Yanagita ET, Alves AK, Oliveira DR. Incidence of regurgitation after the banded gastric bypass. Obes Surg 2005; 15: 1408-1417.

17. Fobi M, Lee H, Igwe D, Felahy B, James E, Stanczyk $\mathrm{M}$, et al. Band erosion: incidence, etiology, management and outcome after banded vertical gastric bypass. Obes Surg 2001; 11: 699-707.

18. Berti LV, Garrido Jr AB. Bypass Gástrico: É essencial a colocação do anel? In: Castro LP, Savassi-Rocha PR, Rodrigues MAG, editores. Tópicos em Gastroenterologia: Obesidade e Urgências Gastroenterológicas. Rio de Janeiro: Medsi; 2003. p. 95-108.

19. Rocha LCM, Lima Jr GF, Martins da Costa MEVM, Girundi MG, Farah MW. A Endoscopia em pacientes submetidos à cirurgia de Fobi-Capella - Análise retrospectiva de 800 exames. GED 2004; 23: 195-204.

20. Galvão Neto MP, Campos JM, Garrido T, Evangelista LF. Migración del anillo después del bypass gástrico. En: Campos JM, Galvão Neto MP, Moura EGH (Org.). Endoscopia en cirugía de la obesidad. 1a ed. Caracas: AMOLCA. p. 181-190. 
21. Huang CS, Farraye FA. Endoscopy in the bariatric surgical patient. Gastroenterol Clin North Am. 2005; 34: 151-166.

22. Campos JM, Evangelista LFL, Siqueira LT, Ferreira Filho HÁ, Ferraz AAB, Ferraz EM. Tratamento endoscópico das complicações do anel no bypass gástrico. In: Anais do Segundo Congresso Latinoamericano International Federation Surgery Obesity-IFSO de Cirurgia para la Obesidad, (Org.). Cacun-México: Colegio Mexicano de Cirugía para la Obesidad 2007. p. 145 .

23. Elias AA, Matielli JD, Oliveira MR, Berti LV, Gabriel J, Szego $T$ et al. Complicações relacionadas com anel de silicone na operação de Capella-Fobi. Bol Cir Obes 2001; 2: 26-26.

24. Campos JM. Tratamento de deslizamento de anel póscirurgia de Fobi-Capella: Técnica de dilatação com balão. In: XVII Seminário Brasileiro de Endoscopia Digestiva e do II Congresso Sudeste de Gastroenterologia. (Org.) SOBED - Sociedade Brasileira de Endoscopia Digestiva; 2005.

25. Garrido T, Maluf Filho F, Sakai P. O Papel da endoscopia na cirurgia bariátrica. In: Garrido Jr. AB, Ferraz EM, Barroso FL, Marchesini JB, Szego T, editores. Cirurgia da obesidade. São Paulo: Atheneu; 2003. p. 293-302.

26. Campos JM, Galvão Neto MP. Tratamento do deslizamento de anel pós-cirurgia de Fobi-Capella. In: Endoscopia gastrointestinal terapêutica. (Org.) SOBED - Sociedade Brasileira de Endoscopia Digestiva. São Paulo: Tecmedd; 2006. p.1143-1147.

27. Taddeucci RJ, Madan AK, Ternovits CA, Tichansky DS. Laparoscopic re-operations for band removal after open banded gastric bypass. Obes Surg 2007; 17: 35 38.

28. Campos JM, Costa Júnior AB, Evangelista LFL. Dificultad de vaciamiento gástrico secundário al anillo. En: Campos JM, Galvão Neto MP, Moura EGH. Endoscopia en cirugía de la obesidad. 1a ed. Caracas: AMOLCA. p.203-213.

29. Francisco MC, Barella SM, Abud TG, Vilar VS, Reibscheid S, Arasaki $\mathrm{CH}$ et al. Análise radiológica das alterações gastrintestinais após cirurgia de Fobi-Capella. Radiol Bras 2007; 40: 235-238. 